\title{
Quasi-arithmetic means and OWA functions in interval-valued and Atanassov's intuitionistic fuzzy set theory
}

\author{
Glad Deschrijver \\ Department of Applied Mathematics and Computer Science, Ghent University, \\ Krijgslaan 281 (S9), B-9000 Gent, Belgium
}

\begin{abstract}
In this paper we propose an extension of the wellknown OWA functions introduced by Yager to interval-valued (IVFS) and Atanassov's intuitionistic (AIFS) fuzzy set theory. We first extend the arithmetic and the quasi-arithmetic mean using the arithmetic operators in IVFS and AIFS theory and investigate under which conditions these means are idempotent. Since on the unit interval the construction of the OWA function involves reordering the input values, we propose a way of transforming the input values in IVFS and AIFS theory to a new list of input values which are now ordered.
\end{abstract}

Keywords: Aggregation function, arithmetic mean, quasi-arithmetic mean, OWA function, interval-valued fuzzy set, Atanassov's intuitionistic fuzzy set

\section{Introduction}

Interval-valued fuzzy set (IVFS) theory $[1,2]$ is an extension of fuzzy theory in which to each element of the universe a closed subinterval of the unit interval is assigned which approximates the unknown membership degree. Another extension of fuzzy set theory is intuitionistic fuzzy set (AIFS) theory introduced by Atanassov [3]. In [4] it is shown that the underlying lattice of Atanassov's intuitionistic fuzzy set (AIFS) theory is the same as for intervalvalued fuzzy set theory and that both are equivalent to $L$-fuzzy set theory in the sense of Goguen [5] w.r.t. a special lattice $\mathcal{L}^{I}$.

OWA functions were introduced by Yager [6] and are used in a variety of applications and studied by many researchers $[7,8,9,10]$. In this paper we propose a generalization of the OWA functions to IVFS and AIFS theory. Several attempts to generalize the OWA function to IVFS or AIFS theory can be found in the literature. $\mathrm{Xu}[11,12]$ and Wei [13] extended the OWA function and related aggregation functions to AIFS theory using a score and accuracy function. This generalization however has several flaws: the aggregation functions based on the total order defined using the score and accuracy function is not monotonous w.r.t. that order; furthermore, these aggregation functions are not consistent with the corresponding aggregation functions on the unit interval. Yager [14] introduced a componentwise extension of the OWA function to AIFS theory, but he gave no motivation why this is the best construction of an OWA function in AIFS theory. Beliakov et al. [15] generalized the construction of $\mathrm{Xu}$ and Wei using additive generators and characterized the functions obtained by the generalized construction which are consistent with the operations on the unit interval. Since the definition of the OWA function on the unit interval involves arithmetic operators on the set of reals, we start in this paper from arithmetic operators on the underlying lattice $\mathcal{L}^{I}$ of IVFS and AIFS theory and we investigate which kind of OWA functions on $\mathcal{L}^{I}$ that we can construct using them. We first recall in Section 2 and 3 some definitions that will be needed later. We recall the axiomatic definition of the arithmetic operators on $\mathcal{L}^{I}$ and we give a characterization of these operators in Section 4 and 5. In the next section we extend the arithmetic mean and the quasi-arithmetic mean to $\mathcal{L}^{I}$ and in the subsequent section we extend the OWA functions to $\mathcal{L}^{I}$. For the latter to be successful we search for a way to extend the ordering procedure of input values in $[0,1]$ to input values in $\mathcal{L}^{I}$.

\section{The lattice $\mathcal{L}^{I}$}

Definition 2.1 We define $\mathcal{L}^{I}=\left(L^{I}, \leq_{L^{I}}\right)$, where

$$
\begin{aligned}
& L^{I}=\left\{\left[x_{1}, x_{2}\right] \mid\left(x_{1}, x_{2}\right) \in[0,1]^{2} \text { and } x_{1} \leq x_{2}\right\}, \\
& {\left[x_{1}, x_{2}\right] \leq_{L^{I}}\left[y_{1}, y_{2}\right] \Longleftrightarrow\left(x_{1} \leq y_{1} \text { and } x_{2} \leq y_{2}\right),} \\
& \quad \text { for all }\left[x_{1}, x_{2}\right],\left[y_{1}, y_{2}\right] \text { in } L^{I} .
\end{aligned}
$$

Similarly as Lemma 2.1 in [4] it can be shown that $\mathcal{L}^{I}$ is a complete lattice.

Definition $2.2[1,2]$ An interval-valued fuzzy set on $U$ is a mapping $A: U \rightarrow L^{I}$.

Definition 2.3 [3] An intuitionistic fuzzy set on $U$ is a set

$$
A=\left\{\left(u, \mu_{A}(u), \nu_{A}(u)\right) \mid u \in U\right\},
$$

where $\mu_{A}(u) \in[0,1]$ denotes the membership degree and $\nu_{A}(u) \in[0,1]$ the non-membership degree of $u$ in $A$ and where for all $u \in U, \mu_{A}(u)+\nu_{A}(u) \leq 1$. 
An intuitionistic fuzzy set $A$ on $U$ can be represented by the $\mathcal{L}^{I}$-fuzzy set $A$ given by

$$
\begin{aligned}
A: U & \rightarrow L^{I}: \\
u & \mapsto\left[\mu_{A}(u), 1-\nu_{A}(u)\right],
\end{aligned}
$$

In Figure 1 the set $L^{I}$ is shown. Note that to each element $x=\left[x_{1}, x_{2}\right]$ of $L^{I}$ corresponds a point $\left(x_{1}, x_{2}\right) \in \mathbb{R}^{2}$.

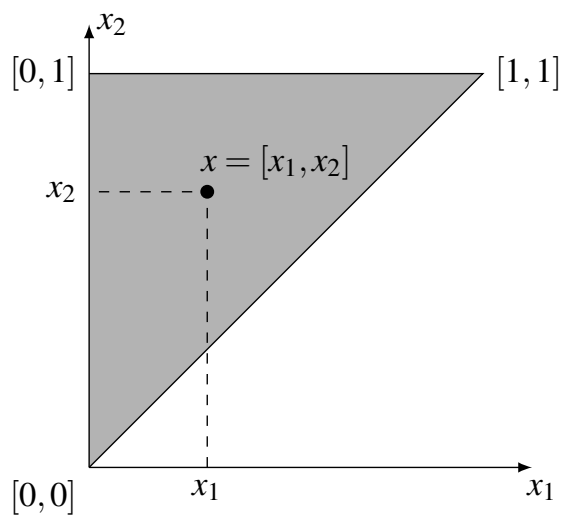

Figure 1: The grey area is $L^{I}$.

In the sequel, if $x \in L^{I}$, then we denote its bounds by $x_{1}$ and $x_{2}$, i.e. $x=\left[x_{1}, x_{2}\right]$. In some occasions, we will also use the notation $x=[\underline{x}, \bar{x}]$. The length $x_{2}-x_{1}$ of the interval $x \in L^{I}$ is called the degree of uncertainty and is denoted by $x_{\pi}$. The smallest and the largest element of $\mathcal{L}^{I}$ are given by $0_{\mathcal{L}^{I}}=[0,0]$ and $1_{\mathcal{L}^{I}}=[1,1]$. Note that, for $x, y$ in $L^{I}, x<_{L^{I}} y$ is equivalent to $x \leq_{L^{I}} y$ and $x \neq y$, i.e. either $x_{1}<y_{1}$ and $x_{2} \leq y_{2}$, or $x_{1} \leq y_{1}$ and $x_{2}<y_{2}$. We define for further usage the set $D=\left\{\left[x_{1}, x_{1}\right] \mid x_{1} \in[0,1]\right\}$.

\section{Aggregation functions on $\mathcal{L}^{I}$}

We denote from now on by $\mathbb{N}^{*}$ the set $\mathbb{N} \backslash\{0\}$. In fuzzy set theory, aggregation functions are defined as follows (see e.g. [16, 17, 18]).

Definition 3.1 An aggregation function $A$ on $[0,1]$ is a mapping $A: \bigcup_{n \in \mathbb{N}^{*}}[0,1]^{n} \rightarrow[0,1]$ with the following properties:

(a1) $A(x)=x$, for all $x \in[0,1]$;

(a2) if $x_{i} \leq y_{i}$ for all $i \in\{1,2, \ldots, n\}$, then $A\left(x_{1}, \ldots, x_{n}\right) \leq A\left(y_{1}, \ldots, y_{n}\right)$, for all $n \in$ $\mathbb{N}^{*}$ and for all $\left(x_{1}, \ldots, x_{n}\right),\left(y_{1}, \ldots, y_{n}\right)$ in $[0,1]^{n}$

(a3) $A(\underbrace{0, \ldots, 0}_{n \text { times }})=0$, for all $n \in \mathbb{N}^{*}$;

(a4) $A(\underbrace{1, \ldots, 1}_{n \text { times }})=1$, for all $n \in \mathbb{N}^{*}$.

This definition is extended to interval-valued fuzzy set theory as follows.
Definition 3.2 [19] An aggregation function $\mathcal{A}$ on $\mathcal{L}^{I}$ is a mapping $\mathcal{A}: \bigcup_{n \in \mathbb{N}^{*}}\left(L^{I}\right)^{n} \rightarrow L^{I}$ with the following properties:

(A1) $\mathcal{A}(x)=x$, for all $x \in L^{I}$;

(A2) if $x_{i} \leq_{L^{I}} y_{i}$ for all $i \in\{1,2, \ldots, n\}$, then $\mathcal{A}\left(x_{1}, \ldots, x_{n}\right) \leq_{L^{I}} \mathcal{A}\left(y_{1}, \ldots, y_{n}\right)$, for all $n \in$ $\mathbb{N}^{*}$ and for all $\left(x_{1}, \ldots, x_{n}\right),\left(y_{1}, \ldots, y_{n}\right)$ in $\left(L^{I}\right)^{n}$

(A3) $\mathcal{A}(\underbrace{0_{\mathcal{L}^{I}}, \ldots, 0_{\mathcal{L}^{I}}}_{n \text { times }})=0_{\mathcal{L}^{I}}$, for all $n \in \mathbb{N}^{*}$;

(A4) $\mathcal{A}(\underbrace{1_{\mathcal{L}^{I}, \ldots, 1_{\mathcal{L}^{I}}},{ }^{n}}_{n \text { times }}=1_{\mathcal{L}^{I}}$, for all $n \in \mathbb{N}^{*}$.

Let $\mathcal{A}$ be an aggregation function on $\mathcal{L}^{I}$ and $\mathcal{N}$ an involutive negator on $\mathcal{L}^{I}$. The mapping $\mathcal{A}^{*}$ : $\bigcup_{n \in \mathbb{N}^{*}}\left(L^{I}\right)^{n} \rightarrow L^{I}$ defined by

$$
\mathcal{A}^{*}\left(x_{1}, \ldots, x_{n}\right)=\mathcal{N}\left(\mathcal{A}\left(\mathcal{N}\left(x_{1}\right), \ldots, \mathcal{N}\left(x_{n}\right)\right)\right),
$$

for all $n \in \mathbb{N}^{*}$ and $x_{1}, \ldots, x_{n}$ in $L^{I}$, is an aggregation function on $\mathcal{L}^{I}$, called the dual aggregation function of $\mathcal{A}$ w.r.t. $\mathcal{N}$.

Aggregation functions on $\mathcal{L}^{I}$ can be constructed in the following way. Let $A_{1}$ and $A_{2}$ be aggregation functions on the unit interval. Define the mapping $\mathcal{A}: \bigcup_{n \in \mathbb{N}^{*}}\left(L^{I}\right)^{n} \rightarrow L^{I}$ by

$$
\begin{aligned}
& \mathcal{A}\left(x_{1}, \ldots, x_{n}\right)=\left[A_{1}\left(\left(x_{1}\right)_{1}, \ldots,\left(x_{n}\right)_{1}\right),\right. \\
& \left.A_{2}\left(\left(x_{1}\right)_{2}, \ldots,\left(x_{n}\right)_{2}\right)\right] \text {, }
\end{aligned}
$$

for all $n \in \mathbb{N}^{*}$ and $x_{1}=\left[\left(x_{1}\right)_{1},\left(x_{1}\right)_{2}\right], \ldots, x_{n}=$ $\left[\left(x_{n}\right)_{1},\left(x_{n}\right)_{2}\right]$ in $L^{I}$. Then $\mathcal{A}$ is an aggregation function on $\mathcal{L}^{I}$ if and only if $A_{1}$ and $A_{2}$ are aggregation functions on $[0,1]$ and $A_{1} \leq A_{2}$.

Not all aggregation functions on $L^{*}$ can be constructed in this way. Consider for example the mapping $\mathcal{A}: \bigcup_{n \in \mathbb{N}^{*}}\left(L^{*}\right)^{n} \rightarrow L^{*}$ defined by, for any aggregation function $A$ on $[0,1]$, for all $n \in \mathbb{N}^{*}$ and $x_{1}, \ldots, x_{n} \in L^{*}$,

$$
\begin{gathered}
\mathcal{A}\left(x_{1}, \ldots, x_{n}\right)=\left[A\left(\left(x_{1}\right)_{1}, \ldots,\left(x_{n}\right)_{1}\right),\right. \\
\max \left(A\left(\left(x_{1}\right)_{1},\left(x_{2}\right)_{2}, \ldots,\left(x_{n}\right)_{2}\right),\right. \\
A\left(\left(x_{1}\right)_{2},\left(x_{2}\right)_{1},\left(x_{3}\right)_{2}, \ldots,\left(x_{n}\right)_{2}\right), \\
\quad \ldots \\
\left.\left.A\left(\left(x_{1}\right)_{2}, \ldots,\left(x_{n-1}\right)_{2},\left(x_{n}\right)_{1}\right)\right)\right]
\end{gathered}
$$

if $n>1$, and $\mathcal{A}\left(x_{1}\right)=\left[A\left(\left(x_{1}\right)_{1}\right), A\left(\left(x_{1}\right)_{2}\right)\right]$ if $n=1$. Then $\mathcal{A}$ is an aggregation function on $\mathcal{L}^{I}$ which does not belong to the previously mentioned class.

Definition 3.3 An aggregation function $\mathcal{A}$ on $\mathcal{L}^{I}$ is called representable if and only if there exist aggregation functions $A_{1}$ and $A_{2}$ on $[0,1]$ such that

$$
\begin{aligned}
& \mathcal{A}\left(x_{1}, \ldots, x_{n}\right)=\left[A_{1}\left(\left(x_{1}\right)_{1}, \ldots,\left(x_{n}\right)_{1}\right),\right. \\
& \left.A_{2}\left(\left(x_{1}\right)_{2}, \ldots,\left(x_{n}\right)_{2}\right)\right] \text {, }
\end{aligned}
$$

for all $n \in \mathbb{N}^{*}$ and for all $x_{1}=\left[\left(x_{1}\right)_{1},\left(x_{1}\right)_{2}\right], \ldots$, $x_{n}=\left[\left(x_{n}\right)_{1},\left(x_{n}\right)_{2}\right]$ in $L^{I}$. 
Note that if a representable aggregation function $\mathcal{T}$ on $\mathcal{L}^{I}$ is a t-norm, then $\mathcal{T}$ is called t-representable (see e.g. $[20,21])$.

Definition 3.4 [19] Let $n \in \mathbb{N} \backslash\{0,1\}$. An $n$ ary aggregation function $\mathcal{A}$ on $\mathcal{L}^{I}$ is a mapping $\mathcal{A}:\left(L^{I}\right)^{n} \rightarrow L^{I}$ with the following properties:

(A1') if $x_{i} \leq_{L^{I}} y_{i}$ for all $i \in\{1,2, \ldots, n\}$, then $\mathcal{A}\left(x_{1}, \ldots, x_{n}\right) \leq_{L^{I}} \mathcal{A}\left(y_{1}, \ldots, y_{n}\right)$, for all $\left(x_{1}, \ldots, x_{n}\right),\left(y_{1}, \ldots, y_{n}\right)$ in $\left(L^{I}\right)^{n}$;

(A2') $\mathcal{A}(\underbrace{0_{\mathcal{L}^{I}}, \ldots, 0_{\mathcal{L}^{I}}}_{n \text { times }})=0_{\mathcal{L}^{I}}$

(A3') $\mathcal{A}(\underbrace{1_{\mathcal{L}^{I}, \ldots, 1_{\mathcal{L}^{I}}}}_{n \text { times }})=1_{\mathcal{L}^{I}}$.

Note that any t-norm or t-conorm on $\mathcal{L}^{I}$ is a binary aggregation function.

If $\mathcal{A}$ is an aggregation function on $\mathcal{L}^{I}$, then the mapping $\mathcal{A}_{n}:\left(L^{I}\right)^{n} \rightarrow L^{I}$ defined by $\mathcal{A}_{n}\left(x_{1}, \ldots\right.$, $\left.x_{n}\right)=\mathcal{A}\left(x_{1}, \ldots, x_{n}\right)$, for all $\left(x_{1}, \ldots, x_{n}\right) \in\left(L^{I}\right)^{n}$ is an $n$-ary aggregation function on $\mathcal{L}^{I}$. Conversely, if for all $n \in \mathbb{N}^{*}, \mathcal{A}_{n}$ is an $n$-ary aggregation function on $\mathcal{L}^{I}$, then the mapping $\mathcal{A}: \bigcup_{n \in \mathbb{N}^{*}}\left(L^{I}\right)^{n} \rightarrow L^{I}$ defined by $\mathcal{A}\left(x_{1}, \ldots, x_{n}\right)=\mathcal{A}_{n}\left(x_{1}, \ldots, x_{n}\right)$, for all $\left(x_{1}, \ldots, x_{n}\right) \in\left(L^{I}\right)^{n}$, for all $n \in \mathbb{N}^{*}$, is an aggregation function on $\mathcal{L}^{I}$.

Example 3.1 Some examples of binary aggregation functions on $\mathcal{L}^{I}$ are, for $x, y$ in $L^{I}$ :

(i) $\mathcal{A}_{A_{1}, A_{2}}(x, y)=\left[A_{1}\left(x_{1}, y_{1}\right), \max \left(A_{2}\left(x_{1}, y_{2}\right)\right.\right.$, $\left.\left.A_{2}\left(y_{1}, x_{2}\right)\right)\right]$

(ii) $\mathcal{A}_{A_{1}, A_{2}}^{*}(x, y)=\left[\min \left(A_{1}\left(x_{1}, y_{2}\right), A_{1}\left(y_{1}, x_{2}\right)\right)\right.$, $\left.A_{2}\left(x_{2}, y_{2}\right)\right]$

(iii) $\mathcal{A}_{A_{1}, A_{2}}^{* *}(x, y)=\left[\min \left(A_{1}\left(x_{1}, y_{2}\right), A_{1}\left(y_{1}, x_{2}\right)\right)\right.$, $\left.\max \left(A_{2}\left(x_{1}, y_{2}\right), A_{2}\left(y_{1}, x_{2}\right)\right)\right]$,

(iv) $\mathcal{A}_{A_{1}, A_{2}, A_{3}, A_{4}}(x, y)=\left[A_{3}\left(A_{1}\left(x_{1}, y_{2}\right)\right.\right.$, $\left.\left.A_{1}\left(y_{1}, x_{2}\right)\right), A_{4}\left(A_{2}\left(x_{1}, y_{2}\right), A_{2}\left(y_{1}, x_{2}\right)\right)\right]$,

where $A_{1}, A_{2}, A_{3}$ and $A_{4}$ are aggregation functions on $[0,1]$ satisfying $A_{1} \leq A_{2}$ and $A_{3} \leq A_{4}$.

Definition 3.5 Consider an aggregation function $\mathcal{A}: \bigcup_{n \in \mathbb{N}^{*}}\left(L^{I}\right)^{n} \rightarrow L^{I}$. Then $\mathcal{A}$ is called idempotent whenever $\mathcal{A}(\underbrace{x, \ldots, x}_{n \text { times }})=x$, for all $n \in \mathbb{N}^{*}$ and $x \in L^{I}$

\section{Arithmetic operators on $\mathcal{L}^{I}$}

Since we want to extend the OWA operators [6] to $\mathcal{L}^{I}$, we need arithmetic operators on $\mathcal{L}^{I}$ (or a superset of $\mathcal{L}^{I}$ ). We define for further usage the sets

$$
\begin{aligned}
& \bar{L}^{I}=\left\{\left[x_{1}, x_{2}\right] \mid\left(x_{1}, x_{2}\right) \in \mathbb{R}^{2} \text { and } x_{1} \leq x_{2}\right\}, \\
& \bar{D}=\left\{\left[x_{1}, x_{1}\right] \mid x_{1} \in \mathbb{R}\right\} ; \\
& \bar{L}_{+}^{I}=\left\{\left[x_{1}, x_{2}\right] \mid\left(x_{1}, x_{2}\right) \in[0,+\infty]^{2}\right.\left.\quad \text { and } x_{1} \leq x_{2}\right\} \\
& \bar{D}_{+}=\left\{\left[x_{1}, x_{1}\right] \mid x_{1} \in[0,+\infty[\},\right.
\end{aligned}
$$

In general we consider two arithmetic operators $\oplus:\left(\bar{L}^{I}\right)^{2} \rightarrow \bar{L}^{I}$ and $\otimes:\left(\bar{L}_{+}^{I}\right)^{2} \rightarrow \bar{L}^{I}$ satisfying the following properties,

$(\mathrm{ADD}-1) \oplus$ is commutative,

$(\mathrm{ADD}-2) \oplus$ is associative,

$(\mathrm{ADD}-3) \oplus$ is increasing,

(ADD-4) $0_{\mathcal{L}^{I}} \oplus a=a$, for all $a \in \bar{L}^{I}$,

$(\mathrm{ADD}-5)[\alpha, \alpha] \oplus[\beta, \beta]=[\alpha+\beta, \alpha+\beta]$, for all $\alpha, \beta$ in $\mathbb{R}$,

$(\mathrm{MUL}-1) \otimes$ is commutative,

$($ MUL-2) $\otimes$ is associative,

$(\mathrm{MUL}-3) \otimes$ is increasing,

(MUL-4) $1_{\mathcal{L}^{I}} \otimes a=a$, for all $a \in \bar{L}_{+}^{I}$,

(MUL-5) $[\alpha, \alpha] \otimes[\beta, \beta]=[\alpha \beta, \alpha \beta]$, for all $\alpha, \beta$ in $[0,+\infty[$.

The conditions (ADD-1)-(ADD-4) and (MUL-1)(MUL-4) are natural conditions for any addition and multiplication operators. The conditions (ADD-5) and (MUL-5) ensure that these operators are natural extensions of the addition and multiplication of real numbers to $\bar{L}^{I}$.

In [22] it was incorrectly stated that (ADD-5) is given by " $[\alpha, \alpha] \oplus[\beta, \beta]=[\alpha+\beta, \alpha+\beta]$, for all $\alpha, \beta$ in $[0,+\infty["$. However, the latter condition together with the condition " $[\alpha, \alpha] \oplus[-\alpha,-\alpha]=0_{\mathcal{L}^{I}}$, for all $\alpha \in[0,+\infty[$ " is equivalent to (ADD-5). Similarly, (MUL-5) can be weakened to " $[\alpha, \alpha] \otimes[\beta, \beta]=$ $[\alpha \beta, \alpha \beta]$, for all $\alpha, \beta$ in $[1,+\infty$ [ or for all $\alpha \in[1,+\infty$ [ and $\beta=\frac{1}{\alpha}$, or for $\alpha=0$ and for all $\beta \in[0,+\infty["$.

Sometimes we will assume that $\oplus$ and $\otimes$ satisfy the following conditions instead of (ADD-5) and (MUL-5):

$\left(\mathrm{ADD}-5^{\prime}\right)[\alpha, \alpha] \oplus b=\left[\alpha+b_{1}, \alpha+b_{2}\right]$, for all $\alpha \in \mathbb{R}$ and $b \in \bar{L}^{I}$

$\left(\mathrm{MUL}^{\prime} 5^{\prime}\right)[\alpha, \alpha] \otimes b=\left[\alpha b_{1}, \alpha b_{2}\right]$, for all $\alpha \in[0,+\infty[$ and $b \in \bar{L}_{+}^{I}$.

These conditions ensure that adding or multiplying an interval with an exact element (an interval with only one element, in other words an interval which does not contain any uncertainty) does not modify the amount of uncertainty in the interval.

The mapping $\ominus$ is defined in [22] by, for all $x, y$ in $\bar{L}^{I}$,

$$
\begin{aligned}
1_{\mathcal{L}^{I}} \ominus x & =\left[1-x_{2}, 1-x_{1}\right], \\
x \ominus y & =1_{\mathcal{L}^{I}} \ominus\left(\left(1_{\mathcal{L}^{I}} \ominus x\right) \oplus y\right) .
\end{aligned}
$$

Similarly, the mapping $\oslash$ is defined by, for all $x, y$ in $\bar{L}_{+, 0}^{I}$,

$$
\begin{aligned}
1_{\mathcal{L}^{I}} \oslash x & =\left[\frac{1}{x_{2}}, \frac{1}{x_{1}}\right], \\
x \oslash y & =1_{\mathcal{L}^{I}} \oslash\left(\left(1_{\mathcal{L}^{I}} \oslash x\right) \otimes y\right) .
\end{aligned}
$$
[22].

The properties of these operators are studied in

Example 4.1 We give some examples of arithmetic operators satisfying the conditions (ADD-1)(ADD-5) and (MUL-1)-(MUL-5). 
- In the interval calculus (see e.g. [23]) the following operators are defined: for all $x, y$ in $\bar{L}^{I}$,

$$
\begin{aligned}
& x \oplus y=\left[x_{1}+y_{1}, x_{2}+y_{2}\right], \\
& x \ominus y=\left[x_{1}-y_{2}, x_{2}-y_{1}\right], \\
& x \otimes y=\left[x_{1} y_{1}, x_{2} y_{2}\right], \quad \text { if } x, y \text { in } \bar{L}_{+}^{I}, \\
& x \oslash y=\left[\frac{x_{1}}{y_{2}}, \frac{x_{2}}{y_{1}}\right], \quad \text { if } x, y \text { in } \bar{L}_{+, 0}^{I} .
\end{aligned}
$$

It is easy to see that these operators satisfy (ADD-1)-(ADD-5), (MUL-1)-(MUL-5), (ADD-5'), (MUL-5'), (1), (2), (3) and (4).

- In [24] the following operators are defined: for all $x, y$ in $\bar{L}^{I}$,

$$
\begin{gathered}
x \oplus_{L^{I}} y=\left[\min \left(x_{1}+y_{2}, x_{2}+y_{1}\right), x_{2}+y_{2}\right], \\
x \ominus_{L^{I}} y=\left[x_{1}-y_{2}, \max \left(x_{1}-y_{1}, x_{2}-y_{2}\right)\right], \\
x \otimes_{L^{I}} y=\left[x_{1} y_{1}, \max \left(x_{1} y_{2}, x_{2} y_{1}\right)\right], \\
\quad \text { if } x, y \text { in } \bar{L}_{+}^{I}, \\
x \oslash_{L^{I}} y=\left[\min \left(\frac{x_{1}}{y_{1}}, \frac{x_{2}}{y_{2}}\right), \frac{x_{2}}{y_{1}}\right], \\
\text { if } x, y \text { in } \bar{L}_{+, 0}^{I} .
\end{gathered}
$$

It was proven in [24] that these operators satisfy (ADD-1)-(ADD-5), (MUL-1)-(MUL-5), (ADD5'), (MUL-5'), (1), (2), (3) and (4). In [25] these operators are used to define additive and multiplicative generators on $\mathcal{L}^{I}$ and it is shown that the only t-norms that can have a continuous additive generator based on this addition are pseudo-t-representable t-norms.

\section{Characterization of the arithmetic operators on $\bar{L}^{I}$}

In this section we give a characterization of the addition operators that satisfy (ADD-1)-(ADD-5) and (ADD-5').

We define the set $\bar{D}^{\prime}=\left\{\left[-x_{2}, x_{2}\right] \mid x_{2} \in\right.$ $\left[0,+\infty[\}\right.$ and the mapping $d: \bar{L}^{I} \rightarrow \bar{D}^{\prime}$ by $d(x)=$ $\left[\frac{x_{1}-x_{2}}{2}, \frac{x_{2}-x_{1}}{2}\right]$, for all $x \in \bar{L}^{I}$.

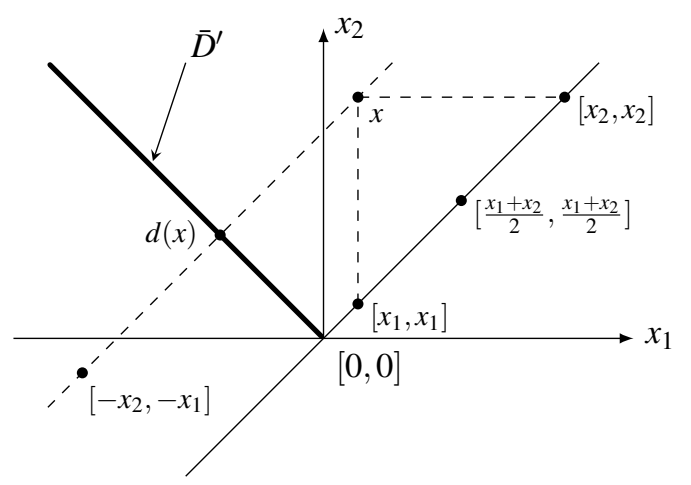

Figure 2: The elements $d(x)$ and $\left[\frac{x_{1}+x_{2}}{2}, \frac{x_{1}+x_{2}}{2}\right]$.

Theorem 5.1 Let $\oplus_{D}:\left(\bar{D}^{\prime}\right)^{2} \cup\left(\bar{D} \times \bar{L}^{I}\right) \cup\left(\bar{L}^{I} \times\right.$ $\bar{D}) \rightarrow \bar{L}^{I}$ be a mapping which satisfies the following conditions:
(D1) $\oplus_{D}$ is commutative,

(D2) for any $x, y, z$ in $\bar{D}^{\prime}, x \oplus_{D} y \leq_{L^{I}}\left(x \oplus_{D} z\right) \oplus_{D}$ $\left[\left|y_{2}-z_{2}\right|,\left|y_{2}-z_{2}\right|\right]$

(D3) for any $x=\left[x_{1}, x_{1}\right] \in \bar{D}$ and $y \in \bar{L}^{I}, x \oplus_{D} y=$ $y \oplus_{D} x=\left[x_{1}+y_{1}, x_{1}+y_{2}\right]$.

Define a mapping $\oplus:\left(\bar{L}^{I}\right)^{2} \rightarrow \bar{L}^{I}$ by, for all $x, y$ in $\bar{L}^{I}$,

$$
\begin{aligned}
& x \oplus y \\
& =\left\{\begin{array}{cc}
x \oplus_{D} y, \quad \text { if }(x, y) \in\left(\bar{D}^{\prime}\right)^{2} \\
\cup\left(\bar{D} \times \bar{L}^{I}\right) \cup\left(\bar{L}^{I} \times \bar{D}\right), \\
\left(d(x) \oplus_{D} d(y)\right) \oplus_{D}\left[\frac{x_{1}+x_{2}+y_{1}+y_{2}}{2},\right. \\
\left.\frac{x_{1}+x_{2}+y_{1}+y_{2}}{2}\right], \quad \text { else. }
\end{array}\right.
\end{aligned}
$$

If furthermore

(D4) for all $(x, y, z) \in\left(\bar{D} \cup \bar{D}^{\prime}\right)^{3}, x \oplus(y \oplus z)=$ $(x \oplus y) \oplus z$,

then $\oplus$ satisfies (ADD-1)-(ADD-5) and (ADD-5'). Conversely, if $\oplus:\left(\bar{L}^{I}\right)^{2} \rightarrow \bar{L}^{I}$ satisfies (ADD1)-(ADD-5) and (ADD-5') and $\oplus_{D}$ is defined as $\oplus_{D}=\left.\oplus\right|_{\left(\bar{D}^{\prime}\right)^{2} \cup\left(\bar{D} \times \bar{L}^{I}\right) \cup\left(\bar{L}^{I} \times \bar{D}\right)}$, then $(D 1)-\left(D_{4}\right)$ and (5) are fulfilled.

Moreover, $\oplus$ is continuous iff $\oplus_{D}$ is continuous.

Theorem 5.1 shows that any addition operator $\oplus$ on $\bar{L}^{I}$ which satisfies (ADD-5') is completely determined by its action on $\bar{D}^{\prime}$.

\section{The arithmetic and quasi-arithmetic mean}

In this section we will extend the arithmetic mean and the quasi-arithmetic mean to $\mathcal{L}^{I}$. Before we do so, we first give some additional properties of the arithmetic operators on $\bar{L}^{I}$.

Lemma 6.1 Assume that $\oplus$ and $\otimes$ satisfy (ADD1)-(ADD-5), (MUL-1)-(MUL-5), (ADD-5') and (MUL5 '). If for all $\alpha, \beta$ in $\mathbb{R}$ and $c \in L^{I}$ it holds that

$$
c \otimes([\alpha, \alpha] \oplus[\beta, \beta])=(c \otimes[\alpha, \alpha]) \oplus(c \otimes[\beta, \beta]),
$$

then for all $a$ and $b$ in $L^{I}$ it holds that

$$
a \oplus b=\left[a_{1}+b_{1}, a_{2}+b_{2}\right] .
$$

In a completely similar way we obtain the following result.

Lemma 6.2 Assume that $\oplus$ and $\otimes$ satisfy (ADD1)-(ADD-5), (MUL-1)-(MUL-5), (ADD-5') and (MUL$\left.5^{\prime}\right)$. If for all $\alpha, \beta$ in $\mathbb{R}$ and $c \in \bar{L}_{+}^{I}$ it holds that

$$
c \otimes([\alpha, \alpha] \oplus[\beta, \beta])=(c \otimes[\alpha, \alpha]) \oplus(c \otimes[\beta, \beta]),
$$

then for all $a$ and $b$ in $\bar{L}_{+}^{I}$ it holds that

$$
a \oplus b=\left[a_{1}+b_{1}, a_{2}+b_{2}\right] .
$$


Theorem 6.3 Assume that $\oplus$ and $\otimes$ satisfy (ADD1) -(ADD-5), (MUL-1)-(MUL-5), (ADD-5') and (MUL$\left.5^{\prime}\right)$. For all $a, b$ in $\mathbb{R}$ and $c \in L^{I}$ it holds that

$$
c \otimes([a, a] \oplus[b, b])=(c \otimes[a, a]) \oplus(c \otimes[b, b]),
$$

iff for all $a$ and $b$ in $L^{I}$ it holds that

$$
a \oplus b=\left[a_{1}+b_{1}, a_{2}+b_{2}\right] .
$$

A similar result holds for $\bar{L}^{I}$. Furthermore:

Theorem 6.4 Assume that $\oplus$ and $\otimes$ satisfy (ADD1) -(ADD-5), (MUL-1)-(MUL-5), (ADD-5') and (MUL$\left.5^{\prime}\right)$. For all $a, b$ in $\mathbb{R}$ and $c \in \bar{L}_{+}^{I}$ it holds that

$$
c \otimes([a, a] \oplus[b, b])=(c \otimes[a, a]) \oplus(c \otimes[b, b]),
$$

iff for all $a$ and $b$ in $\bar{L}^{I}$ it holds that

$$
a \oplus b=\left[a_{1}+b_{1}, a_{2}+b_{2}\right] .
$$

Lemma 6.5 Assume that $\oplus$ and $\otimes$ satisfy (ADD1) -(ADD-5), (MUL-1)-(MUL-5), (ADD-5') and (MUL5'). If for all $n \in \mathbb{N}^{*}$ and $x \in \bar{L}^{I}$ it holds that

$$
[n, n] \otimes x=\underbrace{x \oplus \ldots \oplus x}_{n \text { times }}
$$

then for all $a$ and $b$ in $\bar{L}^{I}$ and $n \in \mathbb{N}$ it holds that

$$
([n, n] \otimes a) \oplus([n, n] \otimes b)=[n, n] \otimes(a \oplus b) .
$$

Lemma 6.6 Assume that $\oplus$ and $\otimes$ satisfy (ADD1) -(ADD-5), (MUL-1)-(MUL-5), (ADD-5') and (MUL5). If for a certain $n \in \mathbb{N}^{*}$ and $x \in \bar{L}^{I}$ it holds that

$$
[n, n] \otimes x=\underbrace{x \oplus \ldots \oplus x}_{n \text { times }}
$$

then also

$$
[n, n] \otimes d(x)=\underbrace{d(x) \oplus \ldots \oplus d(x)}_{n \text { times }},
$$

where $d$ is the $\bar{L}^{I} \rightarrow \bar{D}^{\prime}$ mapping defined in Section 5.

Lemma 6.7 Assume that $\oplus$ and $\otimes$ satisfy (ADD1) -(ADD-5), (MUL-1)-(MUL-5), (ADD-5') and (MUL5 '). For all $n \in \mathbb{N}^{*}$ and $x \in \bar{L}^{I}$ it holds that

$$
[n, n] \otimes x=\underbrace{x \oplus \ldots \oplus x}_{n \text { times }},
$$

if and only if

$$
[n, n] \otimes d(x)=\underbrace{d(x) \oplus \ldots \oplus d(x)}_{n \text { times }} .
$$

Theorem 6.8 Assume that $\oplus$ and $\otimes$ satisfy (ADD1) -(ADD-5), (MUL-1)-(MUL-5), (ADD-5') and (MUL5'). If for all $n \in \mathbb{N}^{*}$ and $x \in \bar{L}^{I} \quad\left(\right.$ or $x \in \bar{L}_{+}^{I}$ ) it holds that

$$
[n, n] \otimes x=\underbrace{x \oplus \ldots \oplus x}_{n \text { times }}
$$

then

$$
x \oplus y=\left[x_{1}+y_{1}, x_{2}+y_{2}\right],
$$

for all $x$ and $y$ in $\bar{L}^{I}$.
In a similar way, we obtain the following results.

Theorem 6.9 Let $a$ and $b$ in $\bar{D}$ with $a<_{L^{I}} b$. Assume that $\oplus$ and $\otimes$ satisfy (ADD-1)-(ADD-5), (MUL-1)-(MUL-5), (ADD-5') and (MUL-5'). If for all $n \in \mathbb{N}^{*}$ and $x \in L^{I}$ (resp. $x \in\left\{x \mid x \in \bar{L}^{I}\right.$ and $x \geq_{L^{I}}$ a and $\left.x \leq_{L^{I}} b\right\}$ ) it holds that

$$
[n, n] \otimes x=\underbrace{x \oplus \ldots \oplus x}_{n \text { times }}
$$

then

$$
x \oplus y=\left[x_{1}+y_{1}, x_{2}+y_{2}\right],
$$

for all $x$ and $y$ in $L^{I}$ (resp. $\left\{x \mid x \in \bar{L}^{I}\right.$ and $x \geq_{L^{I}} a$ and $\left.x \leq_{L^{I}} b\right\}$ ).

It is clear that for any choice of $\oplus$ and $\otimes$ satisfying (ADD-1)-(ADD-5) and (MUL-1)-(MUL-5) respectively, the arithmetic mean $\mathcal{A M}$ defined by

$$
\mathcal{A M}\left(x_{1}, \ldots, x_{n}\right)=\left[\frac{1}{n}, \frac{1}{n}\right] \otimes\left(x_{1} \oplus \ldots \oplus x_{n}\right),
$$

for all $n \in \mathbb{N}^{*}$ and $x_{1}, \ldots, x_{n}$ in $L^{I}$, is an aggregation function on $\mathcal{L}^{I}$.

Lemma 6.10 The mapping $\mathcal{A M}$ defined above is an idempotent aggregation function on $\mathcal{L}^{I}$ if and only if

$$
[n, n] \otimes x=\underbrace{x \oplus \ldots \oplus x}_{n \text { times }}
$$

for all $n \in \mathbb{N}^{*}$ and $x \in L^{I}$.

Theorem 6.11 The mapping $\mathcal{A M}$ defined above is an idempotent aggregation function on $\mathcal{L}^{I}$ if and only if

$$
x \oplus y=\left[x_{1}+y_{1}, x_{2}+y_{2}\right],
$$

for all $x$ and $y$ in $L^{I}$.

We try to extend the quasi-arithmetic means $[26,27,28]$ to $\mathcal{L}^{I}$. We consider a continuous, strictly monotonic function $f: L^{I} \rightarrow \bar{L}^{I}$ for which the inverse is also strictly monotonic. We explicitly require the monotonicity of the inverse because unlike for $\mathbb{R} \rightarrow \mathbb{R}$ functions this does not follow from the other two conditions. For example, the mapping

$\Phi(x)=\left\{\begin{array}{l}{\left[\sqrt{x_{1}}+\frac{x_{1}-\sqrt{x_{1}}}{1-x_{1}}\left(1-x_{2}\right), x_{2}\right], \text { if } x_{1} \neq 1_{\mathcal{L}^{I}}} \\ 1_{\mathcal{L}^{I}}, \text { if } x=1_{\mathcal{L}^{I}}\end{array}\right.$

is a continuous, increasing permutation of $L^{I}$ for which the inverse is not increasing. On the other hand if the inverse of a continuous, strictly monotonic function $f$ is monotonic, then the type of monotonicity is the same for $f^{-1}$ as for $f$, e.g. if the inverse of a continuous, strictly increasing function is monotonic, then it is also increasing.

Let $n \in \mathbb{N}^{*}$. Define the function

$$
\begin{aligned}
& \mathcal{M}_{f}^{n}\left(x_{1}, \ldots, x_{n}\right) \\
& =f^{-1}\left(\left[\frac{1}{n}, \frac{1}{n}\right] \otimes\left(f\left(x_{1}\right) \oplus \ldots f\left(x_{n}\right)\right)\right),
\end{aligned}
$$

for all $x_{1}, \ldots, x_{n}$ in $L^{I}$. 
Theorem 6.12 Let $f$ be a continuous, strictly monotonic function with strictly monotonic inverse. The mapping $\mathcal{M}_{f}^{n}$ defined above is an aggregation function on $\mathcal{L}^{I}$ if and only if

$$
\begin{aligned}
& {[n, n] \otimes f\left(0_{\mathcal{L}^{I}}\right)=\underbrace{f\left(0_{\mathcal{L}^{I}}\right) \oplus \ldots \oplus f\left(0_{\mathcal{L}^{I}}\right)}_{n \text { times }},} \\
& {[n, n] \otimes f\left(1_{\mathcal{L}^{I}}\right)=\underbrace{f\left(1_{\mathcal{L}^{I}}\right) \oplus \ldots \oplus f\left(1_{\mathcal{L}^{I}}\right)}_{n \text { times }} .}
\end{aligned}
$$

If we require that the quasi-arithmetic means are idempotent, then we obtain the following result.

Theorem 6.13 Let $f$ be a continuous, strictly monotonic function with strictly monotonic inverse. The mapping $\mathcal{M}_{f}^{n}$ defined above is an idempotent aggregation function on $\mathcal{L}^{I}$ if and only if

$$
[n, n] \otimes f(x)=\underbrace{f(x) \oplus \ldots \oplus f(x)}_{n \text { times }},
$$

for all $x \in L^{I}$.

Corollary 6.14 Let $f$ be a continuous, strictly monotonic function with strictly monotonic inverse. If for all $\alpha, \beta$ in $\mathbb{R}$ and $c \in \bar{L}_{+}^{I}$ it holds that

$$
c \otimes([\alpha, \alpha] \oplus[\beta, \beta])=(c \otimes[\alpha, \alpha]) \oplus(c \otimes[\beta, \beta]),
$$

and if range $(f) \subseteq \bar{L}_{+}^{I}$, then the mapping $\mathcal{M}_{f}^{n}$ defined above is an idempotent aggregation function on $\mathcal{L}^{I}$.

Lemma 6.15 Let $f: L^{I} \rightarrow \bar{L}^{I}$ be a continuous, strictly monotonic function with strictly monotonic inverse. Then for all $\alpha \in[0,1]$ it holds that $(f([\alpha, \alpha]))_{1}=(f([\alpha, 1]))_{1}$ and $(f([\alpha, \alpha]))_{2}=$ $(f([0, \alpha]))_{2}$ if $f$ is increasing, or $(f([\alpha, \alpha]))_{1}=$ $(f([0, \alpha]))_{1}$ and $(f([\alpha, \alpha]))_{2}=(f([\alpha, 1]))_{2}$ if $f$ is decreasing.

Corollary 6.16 Let $f: L^{I} \rightarrow \bar{L}^{I}$ be a continuous, strictly monotonic function with strictly monotonic inverse. Then $f([0,1])=\left[\left(f\left(0_{\mathcal{L}^{I}}\right)\right)_{1},\left(f\left(1_{\mathcal{L}^{I}}\right)\right)_{2}\right]$ if $f$ is increasing, and $f([0,1])=\left[\left(f\left(1_{\mathcal{L}^{I}}\right)\right)_{1},\left(f\left(0_{\mathcal{L}^{I}}\right)\right)_{2}\right]$ if $f$ is decreasing.

Corollary 6.17 Let $f: L^{I} \rightarrow \bar{L}^{I}$ be a continuous, strictly monotonic function with strictly monotonic inverse. Then $f$ is a bijection from $L^{I}$ to $\left\{\left[x_{1}, x_{2}\right] \mid\left(x_{1}, x_{2}\right) \in[0,1]^{2}\right.$ and $x_{1} \geq\left(f\left(0_{\mathcal{L}^{I}}\right)\right)_{1}$ and $\left.x_{2} \leq\left(f\left(1_{\mathcal{L}^{I}}\right)\right)_{2}\right]$ and $x_{2} \geq \frac{\left(f\left(1_{\mathcal{L}^{I}}\right)\right)_{2}-\left(f\left(0_{\mathcal{L}^{I}}\right)\right)_{2}}{\left(f\left(1_{\mathcal{L}^{I}}\right)\right)_{1}-\left(f\left(0_{\mathcal{L}^{I}}\right)\right)_{1}}\left(x_{1}-\right.$ $\left.\left.\left(f\left(0_{\mathcal{L}^{I}}\right)\right)_{1}\right)+\left(f\left(0_{\mathcal{L}^{I}}\right)\right)_{2}\right\}$ if $f$ is increasing, and $\left\{\left[x_{1}, x_{2}\right] \mid\left(x_{1}, x_{2}\right) \in[0,1]^{2}\right.$ and $x_{1} \geq\left(f\left(1_{\mathcal{L}^{I}}\right)\right)_{1}$ and $\left.x_{2} \leq\left(f\left(0_{\mathcal{L}^{I}}\right)\right)_{2}\right]$ and $x_{2} \geq \frac{\left(f\left(1_{\mathcal{L}^{I}}\right)\right)_{2}-\left(f\left(0_{\mathcal{L}^{I}}\right)\right)_{2}}{\left(f\left(1_{\mathcal{L}^{I}}\right)\right)_{1}-\left(f\left(0_{\mathcal{L}^{I}}\right)\right)_{1}}\left(x_{1}-\right.$ $\left.\left.\left(f\left(0_{\mathcal{L}^{I}}\right)\right)_{1}\right)+\left(f\left(0_{\mathcal{L}^{I}}\right)\right)_{2}\right\}$ if $f$ is decreasing.

Note that in Corollary 6.17 the range of $f$ is a triangle with corners $f\left(0_{\mathcal{L}^{I}}\right), f\left(1_{\mathcal{L}^{I}}\right)$ and $\left[\left(f\left(0_{\mathcal{L}^{I}}\right)\right)_{1},\left(f\left(1_{\mathcal{L}^{I}}\right)\right)_{2}\right]$ if $f$ is increasing, and $f\left(0_{\mathcal{L}^{I}}\right)$, $f\left(1_{\mathcal{L}^{I}}\right)$ and $\left[\left(f\left(1_{\mathcal{L}^{I}}\right)\right)_{1},\left(f\left(0_{\mathcal{L}^{I}}\right)\right)_{2}\right]$ if $f$ is decreasing.
Theorem 6.18 Let $f: L^{I} \rightarrow \bar{L}^{I}$ be a continuous, strictly monotonic function with strictly monotonic inverse. Then there exist strictly monotonic functions $f_{1}, f_{2}:[0,1] \rightarrow \mathbb{R}$ with the same type of monotonicity as $f$ such that for all $x \in L^{I}$,

$$
f(x)=\left[f_{1}\left(x_{1}\right), f_{2}\left(x_{2}\right)\right],
$$

if $f$ is increasing, and

$$
f(x)=\left[f_{1}\left(x_{2}\right), f_{2}\left(x_{1}\right)\right]
$$

if $f$ is decreasing.

Theorem 6.19 Let $f: L^{I} \rightarrow \bar{L}^{I}$ be a continuous, strictly monotonic function with strictly monotonic inverse and for which $f(D) \subseteq \bar{D}$. Consider the mapping $\mathcal{M}_{f}: \cup_{n \in \mathbb{N}^{*}}\left(L^{I}\right)^{n} \rightarrow L^{I}:\left(x_{1}, \ldots, x_{n}\right) \mapsto$ $\mathcal{M}_{f}^{n}\left(x_{1}, \ldots, x_{n}\right)$, for all $n \in \mathbb{N}^{*}$ and $\left(x_{1}, \ldots, x_{n}\right) \in$ $L^{I}$. Then $\mathcal{M}_{f}$ is an idempotent aggregation function on $\mathcal{L}^{I}$ if and only if

$$
x \oplus y=\left[x_{1}+y_{1}, x_{2}+y_{2}\right],
$$

for all $x$ and $y$ in $\left\{x \mid x \in \bar{L}^{I}\right.$ and $x \geq_{L^{I}} \inf \left(f\left(0_{\mathcal{L}^{I}}\right)\right.$, $\left.f\left(1_{\mathcal{L}^{I}}\right)\right)$ and $\left.x \leq_{L^{I}} \sup \left(f\left(0_{\mathcal{L}^{I}}\right), f\left(1_{\mathcal{L}^{I}}\right)\right)\right\}$.

Theorem 6.18 and Theorem 6.19 show that $\mathcal{M}_{f}$ is an idempotent aggregation function iff $\mathcal{M}_{f}$ is representable. This is one of the aggregation functions which can be obtained with the construction of Beliakov et al. [15]. They showed that in their class of generalizations of the quasi-arithmetic mean to $\mathcal{L}^{I}$ the representable extension is the only one which is compatible with the quasi-arithmetic mean on the unit interval.

\section{The OWA function}

The OWA function on the unit interval is defined as follows.

Definition 7.1 [6] Let $n \in \mathbb{N}^{*}$. For any weight vector $w=\left(w_{1}, \ldots, w_{n}\right) \in[0,1]^{n}$ such that

$$
\sum_{i=1}^{n} w_{i}=1
$$

the ordered weighted averaging (OWA) function $\mathcal{O} \mathcal{W} \mathcal{A}_{w}:[0,1]^{n} \rightarrow[0,1]$ associated with $w$ is defined by

$$
\mathcal{O} \mathcal{W} \mathcal{A}_{w}\left(x_{1}, \ldots, x_{n}\right)=\sum_{i=1}^{n} w_{i} x_{(i)}
$$

where $\left(x_{1}, \ldots, x_{n}\right) \in[0,1]^{n}$ is reordered as $x_{(1)} \leq$ $\ldots \leq x_{(n)}$.

In order to extend the OWA function to intervalvalued fuzzy sets, we must find a way to extend the ordering of input values in $[0,1]$ to input values which are intervals and which therefore may be incomparable. 
If we consider $x_{1}=\left[\underline{x}_{1}, \bar{x}_{1}\right], \ldots, x_{n}=\left[\underline{x}_{n}, \bar{x}_{n}\right]$ in $L^{I}$ as fuzzy truth values $[29,30]$, i.e. as mappings

$$
\begin{aligned}
x_{i}:[0,1] & \rightarrow[0,1]: \\
u & \mapsto 1, \quad \forall u \in\left[\underline{x}_{1}, \bar{x}_{2}\right], \\
u & \mapsto 0, \quad \text { else, }
\end{aligned}
$$

and if we apply Zadeh's extension principle, then we obtain

$$
\begin{aligned}
x_{(i)}(u)= & \sup \left\{\min \left(x_{1}\left(u_{1}\right), \ldots, x_{n}\left(u_{n}\right)\right) \mid\right. \\
& \left.\left(u_{1}, \ldots, u_{n}\right) \in[0,1]^{n} \text { and } u=u_{(i)}\right\} \\
= & {\left[\underline{x}_{(i)}, \bar{x}_{(i)}\right], }
\end{aligned}
$$

where $\underline{x}_{(i)}$ denotes the $i$-th smallest element of $\left\{\underline{x}_{1}, \ldots, \underline{x}_{n}\right\}$ and $\bar{x}_{(i)}$ denotes the $i$-th smallest element of $\left\{\bar{x}_{1}, \ldots, \bar{x}_{n}\right\}$.

Definition 7.2 Let $n \in \mathbb{N}^{*}$. Assume that $\oplus$ and $\otimes$ satisfy (ADD-1)-(ADD-5), (MUL-1)-(MUL-5) and ${ }^{1}$ $0_{\mathcal{L}^{I}} \otimes x=0_{\mathcal{L}^{I}}$ for all $x \in L^{I}$. For any weight vector $w=\left(w_{1}, \ldots, w_{n}\right) \in\left(L^{I}\right)^{n}$ such that

$$
\bigoplus_{i=1}^{n} w_{i}=1_{\mathcal{L}^{I}}
$$

the ordered weighted averaging (OWA) function $\mathcal{O} \mathcal{W A}_{w}:\left(L^{I}\right)^{n} \rightarrow L^{I}$ associated with $w$ is defined by

$$
\mathcal{O} \mathcal{W} \mathcal{A}_{w}\left(x_{1}, \ldots, x_{n}\right)=\bigoplus_{i=1}^{n}\left(w_{i} \otimes x_{(i)}\right)
$$

where $\left(x_{1}, \ldots, x_{n}\right) \in\left(L^{I}\right)^{n}$ is transformed to $x_{(1)} \leq_{L^{I}} \ldots \leq_{L^{I}} x_{(n)}$ using the above construction.

It is easy to see that the OWA function on the unit interval is idempotent. We now check under which conditions the OWA function on $\mathcal{L}^{I}$ is idempotent.

Theorem 7.1 Assume that $\oplus$ is continuous and satisfies (ADD-1)-(ADD-5) and (ADD-5'). Then for all $x$ and $y$ in $\bar{L}^{I}$,

$$
x \oplus y \in \bar{D} \Longleftrightarrow x \in \bar{D} \text { and } y \in \bar{D} .
$$

As a consequence of Theorem 7.1 we have that $\oplus_{i=1}^{n} w_{i}=1_{\mathcal{L}^{I}}$ implies that $w_{i} \in D$ for all $i \in$ $\{1, \ldots, n\}$. In other words, the weights of an OWA function on $\mathcal{L}^{I}$ cannot contain any uncertainty (if a continuous addition operator is used).

We say that a function $f:\left(L^{I}\right)^{n} \rightarrow L^{I}$ is a joinmorphism if for all $k \in\{1, \ldots, n\}$ and $\left(x_{1}, \ldots, x_{k-1}\right.$, $\left.x_{k}, x_{k}^{\prime}, x_{k+1}, \ldots, x_{n}\right) \in[0,1]^{n+1}$ it holds that

$$
\begin{gathered}
f\left(x_{1}, \ldots, x_{k-1}, \sup \left(x_{k}, x_{k}^{\prime}\right), x_{k+1}, \ldots, x_{n}\right) \\
=\sup \left(f\left(x_{1}, \ldots, x_{k-1}, x_{k}, x_{k+1}, \ldots, x_{n}\right),\right. \\
\left.f\left(x_{1}, \ldots, x_{k-1}, x_{k}^{\prime}, x_{k+1}, \ldots, x_{n}\right)\right)
\end{gathered}
$$

If we replace sup by inf in the previous definition, then we obtain the definition of a meet-morphism. $\left.5^{\prime}\right)$.
Theorem 7.2 Let $\mathcal{A}$ be a binary aggregation function on $\mathcal{L}^{I}$ which is idempotent and both a join- and meet-morphism. If for all $x_{1}$ and $x_{2}$ in $[0,1]$ it holds that

$$
\begin{aligned}
& \left(\mathcal{A}\left(\left[x_{1}, 1\right],[0,0]\right)\right)_{1}=\left(\mathcal{A}\left(\left[x_{1}, 1\right],[0,1]\right)\right)_{1}, \\
& \left(\mathcal{A}\left(\left[0, x_{2}\right],[0,1]\right)\right)_{2}=\left(\mathcal{A}\left(\left[0, x_{2}\right],[1,1]\right)\right)_{2},
\end{aligned}
$$

then there exist aggregation operators $A_{1}$ and $A_{2}$ on $([0,1], \leq)$ such that $A_{1} \leq A_{2}$ and

$$
\mathcal{A}(x, y)=\left[A_{1}\left(x_{1}, y_{1}\right), A_{2}\left(x_{2}, y_{2}\right)\right],
$$

for all $x$ and $y$ in $L^{I}$.

It remains an open problem whether Theorem 7.2 can be generalized to $n$-ary aggregation functions with $n>2$.

Define the mapping $\mathcal{O} \mathcal{W} \mathcal{A}_{w}:\left(L^{I}\right)^{n} \rightarrow L^{I}$ by

$$
\begin{aligned}
& \mathcal{O W} \mathcal{A}_{w}\left(x_{1}, \ldots, x_{n}\right) \\
& =\bigoplus_{i=1}^{n}\left(\left[w_{i}, w_{i}\right] \otimes x_{(i)}\right) \\
& =\left[\mathrm{OWA}_{w}\left(\underline{x}_{1}, \ldots, \underline{x}_{n}\right), \mathrm{OWA}_{w}\left(\bar{x}_{1}, \ldots, \bar{x}_{n}\right)\right],
\end{aligned}
$$

for all $\left(x_{1}, \ldots, x_{n}\right) \in\left(L^{I}\right)^{n}$, where $\mathrm{OWA}_{w}$ is the OWA function defined on the unit interval associated with $w=\left(w_{1}, \ldots, w_{n}\right) \in[0,1]^{n}$ with $\sum_{i=1}^{n} w_{i}=1$, and $\oplus$ is the representable addition operator (the first example in Example 4.1). In light of Theorem 6.11, Theorem 6.18 , Theorem 6.19 and Theorem 7.2, we put the conjecture that the only extension of the OWA function to $\mathcal{L}^{I}$ using the arithmetic operators on $\mathcal{L}^{I}$ which is idempotent, is the representable extension $\mathcal{O} \mathcal{W} \mathcal{A}_{w}$. Beliakov et al. [15] have given a similar extension of the OWA function, but instead of extending the ordering mechanism using Zadeh's extension principle, they use a total order based on the score and the accuracy of elements of $\mathcal{L}^{I}$. This extension of the OWA function is not representable. They have shown that in their class of generalizations of the OWA function, this extension is the only one which is consistent with the OWA function on the unit interval.

\section{Conclusion}

In this paper we have extended several well-known aggregation functions to $\mathcal{L}^{I}$, the underlying lattice of interval-valued and Atanassov's intuitionistic fuzzy set theory. First we have shown that under several different conditions the arithmetic operators on $\mathcal{L}^{I}$ are t-representable. This fact has been used to prove that any extension of the arithmetic mean and the quasi-arithmetic mean to $\mathcal{L}^{I}$ using arithmetic operators on $\mathcal{L}^{I}$ is t-representable if and only if it is idempotent. We have in general shown that idempotent binary aggregation functions which are join- and meet-morphisms and which satisfy some additional properties are t-representable. We have extended the ordering procedure of the input values to $\mathcal{L}^{I}$ and given a proposal for the construction of the OWA function on $\mathcal{L}^{I}$. 


\section{References}

[1] M. B. Gorzałczany. A method of inference in approximate reasoning based on intervalvalued fuzzy sets. Fuzzy Sets and Systems, 21(1):1-17, 1987.

[2] R. Sambuc. Fonctions $\Phi$-floues. Application à l'aide au diagnostic en pathologie thyroidienne. PhD thesis, Université de Marseille, France, 1975.

[3] K. T. Atanassov. Intuitionistic fuzzy sets. Physica-Verlag, Heidelberg, New York, 1999.

[4] G. Deschrijver and E. E. Kerre. On the relationship between some extensions of fuzzy set theory. Fuzzy Sets and Systems, 133(2):227235, 2003.

[5] J. A. Goguen. L-fuzzy sets. Journal of Mathematical Analysis and Applications, 18(1):145174, 1967.

[6] R. R. Yager. On ordered weighted averaging aggregation operators in multicriteria decision making. IEEE Trans. Syst. Man. Cybern., 18:183-190, 1988.

[7] J. C. Fodor, J.-L. Marichal, and M. Roubens. Characterization of some aggregation functions arising from mcdm problems. In B. BouchonMeunier, R. R. Yager, and L. A. Zadeh, editors, Fuzzy Logic and Soft Computing, volume 4 of Advances in Fuzzy Systems - Application and Theory, pages 194-201. World Scientific Publishing, Singapore, 1995.

[8] M. Grabisch, J.-L. Marichal, R. Mesiar, and E. Pap. Aggregation functions: means. Information Sciences, 181:1-22, 2011.

[9] M. Grabisch. On equivalence classes of fuzzy connectives: the case of fuzzy integrals. IEEE Transactions on Fuzzy Systems, 3:96109, 1995.

[10] J.-L. Marichal and P. Mathonet. A characterization of the ordered weighted averaging functions based on the ordered bisymmetry property. IEEE Transactions on Fuzzy Systems, $7(1): 93-96,1999$.

[11] Z. Xu. Intuitionistic fuzzy aggregation operators. IEEE Transactions on Fuzzy Systems, 15:1179-1187, 2007.

[12] Z. Xu. Choquet integrals of weighted intuitionistic fuzzy information. Information Sciences, 180:726-736, 2010.

[13] G. Wei. Some induced geometric aggregation operators with intuitionistic fuzzy information and their application to group decision making. Applied Soft Computing, 10:423-431, 2010.

[14] R. R. Yager. Owa aggregation of intuitionistic fuzzy sets. International Journal of General Systems, 38:617-641, 2009.

[15] G. Beliakov, H. Bustince, D. P. Goswami, U. K. Mukherjee, and N. R. Pal. On averaging operators for atanassov's intuitionistic fuzzy sets. Information Sciences, 181:1116-1124, 2011.
[16] T. Calvo, A. Kolesárová, M. Komorníková, and R. Mesiar. Aggregation operators: properties, classes and construction methods. In T. Calvo, G. Mayor, and R. Mesiar, editors, Aggregation Operators: New Trends and Applications, pages 3-104. Physica-Verlag, Heidelberg/New York, 2002.

[17] E. P. Klement, R. Mesiar, and E. Pap. On the relationship of associative compensatory operators to triangular norms and conorms. International Journal of Uncertainty, Fuzziness and Knowledge-Based Systems, 4(2):129-144, 1996.

[18] A. Kolesárová and M. Komorníková. Triangular norm-based iterative compensatory operators. Fuzzy Sets and Systems, 104(1):109-120, 1999.

[19] G. Deschrijver and E. E. Kerre. Implicators based on binary aggregation operators in interval-valued fuzzy set theory. Fuzzy Sets and Systems, 153(2):229-248, 2005.

[20] C. Cornelis, G. Deschrijver, and E. E. Kerre. Implication in intuitionistic and interval-valued fuzzy set theory: construction, classification and application. International Journal of Approximate Reasoning, 35(1):55-95, 2004.

[21] G. Deschrijver, C. Cornelis, and E. E. Kerre. On the representation of intuitionistic fuzzy tnorms and t-conorms. IEEE Transactions on Fuzzy Systems, 12(1):45-61, 2004.

[22] G. Deschrijver. Generalized arithmetic operators and their relationship to t-norms in interval-valued fuzzy set theory. Fuzzy Sets and Systems, 160(21):3080-3102, 2009.

[23] R. E. Moore. Interval arithmetic. PrenticeHall, Englewood Cliffs, NJ, USA, 1966.

[24] G. Deschrijver. Arithmetic operators in interval-valued fuzzy set theory. Information Sciences, 177(14):2906-2924, 2007.

[25] G. Deschrijver. Additive and multiplicative generators in interval-valued fuzzy set theory. IEEE Transactions on Fuzzy Systems, 15(2):222-237, 2007.

[26] A. N. Kolmogorov. Sur la notion de la moyenne. Atti della Reale Accademia Nazionale dei Lincei, 12(6):388-391, 1930.

[27] M. Nagumo. Über eine Klasse der Mittelwerte. Japan Journal of Mathematics, 7:71-79, 1930.

[28] J. Aczél. On mean values. Bulletin of the American Mathematical Society, 54:392-400, 1948.

[29] Z. Gera and J. Dombi. Type-2 implications on non-interactive fuzzy truth values. Fuzzy Sets and Systems, 159:3014-3032, 2008.

[30] C. L. Walker and E. A. Walker. The algebra of fuzzy truth values. Fuzzy Sets and Systems, 149:309-347, 2005. 